\title{
Data Communications Laboratory as a Core Element for Modern Education
}

\author{
Tomáš Zeman, Jaromír Hrad, Jakub Slíva and Jiř́i Hájek \\ Czech Technical University in Prague, Faculty of Electrical Engineering, \\ Department of Telecommunication Engineering \\ Technická 2, CZ-166 27 Praha 6, Czech Republic \\ \{zeman,hrad,slivaj1,hajekj3\}@fel.cvut.cz, http://www.comtel.cz
}

\section{Summary}

The present education in the area of data communications brings one substantial pedagogical dilemma. The given area is purely technical one. Its education inherently includes the contact with teachers during lectures, theoretical seminars, simulations of various processes, real measurements and analyses in a laboratory. Therefore the popular e-learning approaches cannot be fully utilized since that could possibly prevent students' access to laboratory measurements. On the other hand, preserving of fully contact education would be a drawback, perceived negatively by students as well as their teachers.

We were looking for a way to prepare a modern curriculum for education in data communications and we decided for a procedure based on a project of a new Data Communications Laboratory that should become a core element in the modernized pedagogical process.

Modern education clearly requires the following formal components in order to satisfy the needs of all involved parties: data warehouse for storage of lectures (prerecorded audiovisual materials) for flexible use at any time, consulting lectures to clarify difficult parts of the pre-recorded materials, simulations of procedures in data communications supporting remote access and control, workplace for configuration of data communications devices supporting remote access and control, laboratory equipment and workplaces with flexibly scheduled access, set of laboratory tasks, portal for processing of laboratory reports (results, simulations) and testing, including a suitable communication interface, electronic library of supporting materials (textbooks, articles, slides, etc.), control workplace for teachers, research unit for the further development of the respective area. 
Our team has proposed a methodology for education that takes into consideration the implementation of all the above-listed components, forming a compact system. Its technological core is the newly designed Data Communications Laboratory.

The essential requirement for education at a modern university is the independence of the students from any specific time for lessons. This condition determines the need to use a wide spectrum of various distance education forms, especially e-learning ones. This is, however, complicated by the necessity to provide practical education that must be performed in a laboratory. Nevertheless, even such type of education can be optimized for the students in the terms of its proper time scheduling. The solution consists in flexible selection of free time slots in the laboratory that are reserved by the students well in advance or just before the respective measurement.

In the optimized curriculum, the lectures will be pre-recorded and available to students as streamed media from the data warehouse. This way will be used for routine chapters that do not require extensive explanation. More complex topics will be discussed at the scheduled special seminars and/or lectures.

The principal advantage of this solution is that the respective activities are not strictly bound with one specific time (except the advanced lectures). There are defined relatively wide time windows (providing sufficient reserve for a subject covered by two hours of lectures and two hours of exercises weekly) respecting the individual pace of study as well as time possibilities of all students.

It is important to mention the laboratory exercises. While the configuration and simulation tasks (as well as the processing of the project) are performed individually, the laboratory work is a team one. The measuring team consists of three students at most so that the individuality is not suppressed. The composition of the teams may change for every measurement tasks out of the four (in the case of our example) students choose a suitable (and available) time window, without knowing the partners or having a chance to influence their choice. Thanks to this principle, students learn to collaborate within a heterogeneous group.

The present project shows that the modern concept of education cannot be simplified just to e-learning; our attention must be paid to all different aspects of education. 